\title{
Effective "Vaporizer" for recovering retrograde hydrocarbon condensate from a gas-condensate
} reservoir

\begin{abstract}
This paper investigates some fundamental aspects of a "vaporiser" for recovering trapped retrograde condensate in the formation that is formed during exploitation gascondensate reservoirs in depletion regime. The thermodynamic test of seven different gas-condensate systems and analysis of the liquid and gas phase's samples which were taken under the same thermobaric conditions was provided wide information about the occurrences in the reservoir fluids. It was identified that the solubility capability of gas mixture in the hydrocarbon condensate is elevated and improved as a "vaporiser" if it's critical temperature is increased but compressibility factor and critical pressure are decreased. It was determined that, improving the solubility of gas components in the condensate decreases the system fog up and retrograde condensation pressures and improves stability of aerosol condition of gas-condensate fluid. Therefore, injected gas for gas cycling or re-vaporization of condensate from the core can be controlled for the purpose of increasing its solubility.
\end{abstract}

Keywords: gas-condensate, gas injection, fog up pressure, gas solubility, gas solubility, dissolved gas
Volume 2 Issue 6 - 2017

\author{
Vugar M Fataliyev,' Natig N Hamidov² \\ 'National Academy of Sciences of Azerbaijan, Institute Oil and \\ Gas, Azerbaijan \\ ${ }^{2}$ State Oil Company of Azerbaijan Republic (SOCAR), Institute \\ Oil and Gas, Azerbaijan
}

\begin{abstract}
Correspondence: Vugar M Fataliyev, National Academy of Sciences of Azerbaijan, Institute Oil and Gas, Tel 994506834383 , Email fatavm@bp.com
\end{abstract}

Received: May 17, 2017 | Published: July 18, 2017

\section{Introduction}

A significant portion of current hydrocarbon reserves exists in gas/condensate-carrying formations. In analogy to oil reservoirs, the production of condensate fields by pressure depletion only may result in significant loss of the heavy ends owing to liquid dropout below the Retrograde Dew Point (RDP) Pressure. ${ }^{1,2}$ The condensed liquid increases the fluid saturation in the near-wellbore formation and, therefore, has the potential to decrease the gas relative permeability. ${ }^{3}$ The accumulated condensate bank also changes the phase composition, which in turn reshapes the phase diagram and other properties of reservoir fluid. ${ }^{4}$ Results from the case study ${ }^{5-7}$ showed that the impact of condensate banking would be severe for these reservoirs if the physical processes expected in these reservoirs are not correctly modelled.

Unlike others, paper $^{7}$ experimentally proved a significant improvement in gas relative permeability of the condensate reservoir even by injecting methanol, but one of the primary techniques the gas injection method is widely used to eliminate retrograde condensation or to prevent condensate banking in the formation. ${ }^{8,9}$ Constant Composition Expansion (CCE) studies were conducted in the paper $^{10}$ to measure the retrograde liquid deposit data for a real reservoir fluid. Subsequently, the effect of adding light hydrocarbon gases on the vaporization of condensed liquid was studied. Results indicated that carbon dioxide $\left(\mathrm{CO}_{2}\right)$ extracts the condensed liquids in the near-wellbore region and, consequently, increases the productivity upon bringing the well back on-line. In our earlier works ${ }^{1,11}$ modelled revalorization process of hydrocarbon condensate by injecting "dry natural gas" with different composition of carbon dioxide and nitrogen. It has been demonstrated that $\mathrm{CO}_{2}$ gas mixture shows considerable promise as an injected "vaporizer". In contrast, nitrogen has been shown to be unsuitable for the envisioned application. However, the use of $\mathrm{N}_{2}$ may prove economically viable in certain circumstances and can be considered as a potential option to improve productivity.

Generally, as it was re-confirmed in the papers ${ }^{1,8}$ based on evidence that, maintaining gas condensate reservoir pressure above the RDP pressure of the reservoir fluid is the preferable method for gascondensate reservoir development but according to the some other researches ${ }^{4}$ confirmed that, it is possible to maintain reservoir pressure below the RDP pressure whilst reducing the reservoir pressure correspondently, as injected dry gas vaporizes both intermediate and some heavy hydrocarbons, decreases condensate/gas ratio and reduces the RDP pressure. Other facts which were provided in the works ${ }^{12,13}$ proved that development of miscibility in gas cycling schemes may be achieved at pressures far below the dew-point pressure of the condensate by injection of $\mathrm{CO}_{2}$. The failure of lowmiscibility pressure obtained for injection of $\mathrm{CO}_{2}$ in a retrograde condensate suggests that mature condensate carrying formations may be suitable targets for $\mathrm{CO}_{2}$ sequestration offset by a possible increase in condensate recovery.

The experimental investigations of the natural gas-condensate systems at high pressures determine the existence of gas components well-soluble in hydrocarbon condensate in the initial composition of the formation gas results in the decrease of values of dew point and therefore the prolongation of the development period of the deposit in a single-phase gas regime. ${ }^{14}$ Therefore, injected gas or "vaporiser" for gas cycling or re-vaporization of condensate from the core can be controlled for the purpose of increasing its solubility and gascondensate well deliverability accordingly. ${ }^{11,14}$

In point of fact, gas injection method is associated with complex thermodynamic processes or phase transitions, such as the re- 
vaporization of heavy hydrocarbon ends and connate water, the reduction of the condensate/gas ratio and RDP pressure, etc. ${ }^{12,15}$ Nevertheless, successful design and implementation of enhanced condensate recovery schemes require accurate prediction of the compositional effects that control the local vaporization/displacement efficiency. In line with these conclusions, and also results which were obtained in the earlier works ${ }^{4,14,15}$ in this paper, we intended to find out a way of improving the effectiveness of "vaporiser" for the recovering trapped retrograde condensate in the formation, which is left after primary production. To this purpose, this paper performed an experimental investigation into this phenomenon as it is problematic during mathematical modelling.

\section{Investigation method and procedure}

\section{Laboratory apparatuses}

Experiments were carried out on a УГК type of РVТ bomb, which is a standard apparatus for determining thermodynamic characteristics and the phase behaviour of gas condensate systems. ${ }^{1}$ The schematic diagram of the experimental laboratory apparatus and the purposes of the laboratory modules are presented in the Figure 1. The maximum working pressure of the PVT bomb is $45 \mathrm{MPa}$, maximum working temperature is $80^{\circ} \mathrm{C}$ and cell volume is $3 \times 10^{-3} \mathrm{~m}^{3}$. As shown in the diagram Figure 1, the laboratory facility can be divided into 9 Modules:

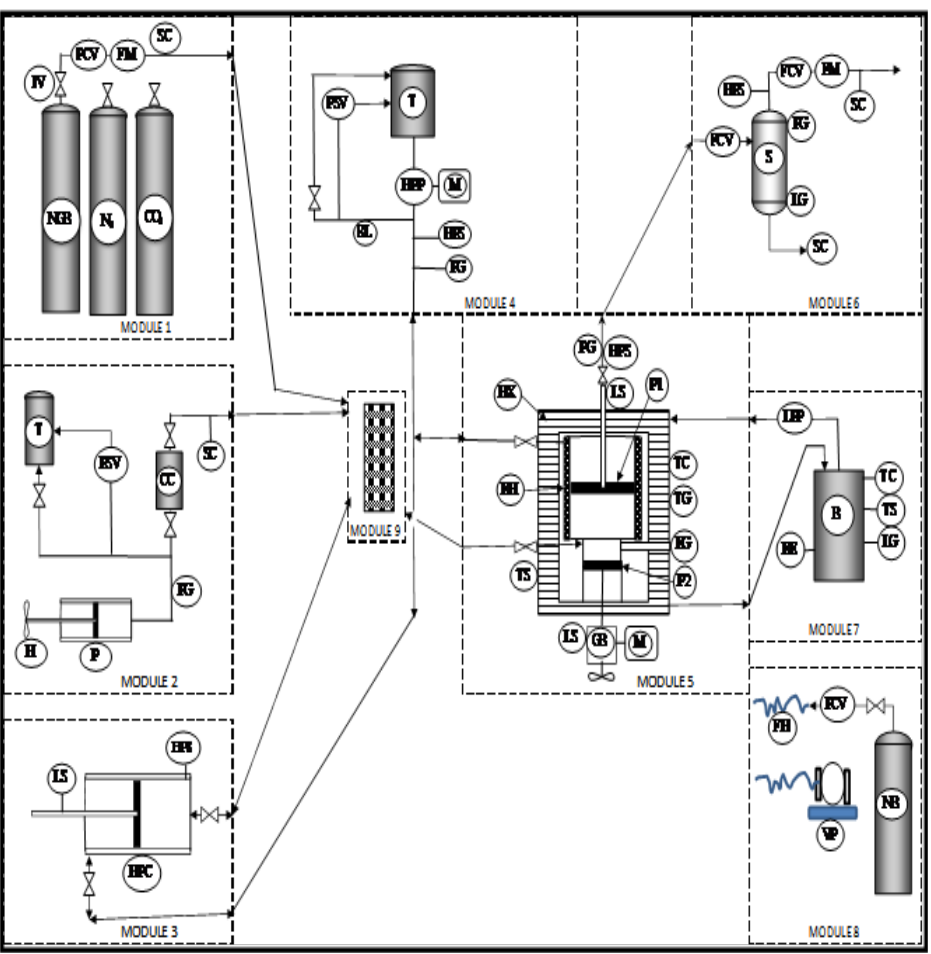

Figure I Schematic representation of laboratory apparatus

Module 1: Module 1 is for handling natural gas from the bottle. It is equipped with Natural Gas Bottle-NGB, $\mathrm{CO}_{2}$ Bottle-CB, Nitrogen Bottle-NB, Pressure Control Valve-PCV, Flow Meter-FM and normal Isolation Valve-IV. Sample Connection-SC allows the provision of sampling for composition analyses. This module can be linked very easily with Modules 3 and 5 by manifold (Module 9) for recombining gas mixture and gas-condensate system.

Module 2: Module 2 is for handling hydrocarbon condensate from the container. It is equipped with Condensate Container-CC, glycerine reservoir or Tank-T and Press-P for squeezing condensate with glycerine. The operator can control and reconfirm the volume of squeezed condensate by using the volume scale and Handle-H. SC is for examining the composition of the condensate sample. Pressure Safety Valve-PSV, IVs and Pressure Gauge-PG are used for performing safe operations. The releasable connection element is constructed in Module 9 for transferring liquid samples from Module 2 to Module 5 for recombining gas-condensate mixture.

Module 3: Module 3 is for performing gas mixture preparation. Hydraulic-driven reciprocating type of High Pressure Compressor-HPC compresses gas (natural, nitrogen or carbon dioxide) and introduces to the PVT cell (Module 5). The High Pressure Pump-HPP in Module 4 supplies hydraulic (glycerine) pressure. The Compressor is equipped with instruments for precisely monitoring volume, temperature and pressure. Limit Switches-LS keeps the piston in the safe travelling limit and High Pressure Switch-HPS trips HPP and maintains safe operation.

Module 4: Module 4 provides high hydraulic (glycerine) pressure for increasing pressure of gas-condensate system in the PVT cell (Module 5) and gas in the Module 3. This Module consists of oil tank (T), electrical Motor-M driven reciprocating type of High Pressure Pump-HPP and safety devices (PSV and HPS). Also, the units (including Module 3 and 5) can be depressurized in a controlled and safe manner via the oil Return Line-RL to the tank.

Module 5: Module 5 is a PVT cell. This facility consists of two components; gas (upper) and condensate (lower) sections. Piston-P1 in the upper section is moved by hydraulic force which Module 4 supplies. The Piston-P2 in the condensate section can be moved by electrical motor $(\mathrm{M})$ or manually using handle $(\mathrm{H})$ via Gear Box-GB for monitoring or measuring liquid level in the section. Instruments on this unit allow for the measurement of cell volume, condensate volume, temperature and pressure. The Condensate level can be monitored by the naked eye through Eyeglass-EG and also can be displayed. There 
are two heat sources. Electrical Heater-EH and Heat Exchanger-HX are mounted on the PVT cell. EH is the main source and equipped with Temperature Gauge-TG and Temperature Controller-TC. The TC takes an input from a temperature sensor and has an output that is connected to a EH. The purpose of HX is to maintain a barrier between PVT Cell and the ambience and minimise heat losses by circulating heat transfer medium (oil) through the plates. Module 7 provides heat transfer medium. PSV and HPSs provide overpressure protection barrier, Temperature Switch-TS protects system from overheating and LSs provide pistons to travel in a safe operating limit.

Module 6: Module 6 is mounted for processing the separation of liquid from gas in the constant operating parameters. PCV and Flow Control Valve-FCV maintain stable operation. There are relevant instruments to indicate pressure, temperature, gas and liquid volume. The liquid level in the separator is monitored through the separator window-level Gauge (LG). Also, a sample connection is designed for regular sampling from gas and liquid phases. HPS protects equipment from over-pressurization.

Module 7: Module 7 is to generate heat and transfer it into Module 5. It consists of EH, Boiler-B with Level Gauge-LG and Low Pressure Pump-LPP. The Electrical Heater generates heat, TC controls temperature at a set point. LPP circulates heated oil (heat transfer medium) through heat exchanger (Module 5). TS protect system from overheating.

Module 8: Module 8 is cleaner. Vacuum Pump-VP and Nitrogen Bottles-NB and Flexible Hoses-FH are used for purging, vacuuming and preparing experimental devices for the next experimental round.

Module 9: Module 9 is manifold. It is constructed with quick connection elements. These connection elements allow the operator to link different modules for loading Module 3 and 5 with NG, recombining gas or gas-condensate mixture in the PVT cell. Also, it can be used for purging and cleaning the laboratory facility.

In addition, the original design of the PVT bomb has been modified slightly within safe operating limits. A heat exchanger was installed to increase working temperature of the bomb up to $110^{\circ} \mathrm{C}$. Recent calibrated gauges and devises were used during experiments, ensuring their accuracies were in the designed limit. Class 1.0 with $\pm 1 \%$ error pressure gauges and temperature gauges with $\pm 0.4^{\circ} \mathrm{C}$ were used.

\section{Gas-condensate sampling}

Our investigation was not intended to study any specific field. The main goal was to examine new ideas, to understand the importance and a role of the solubility of the gas in the phase behaviour of gascondensate systems during reservoir exploitation and the improvement of the gas-condensate recovery method. Therefore, it was not necessary for the samples to replicate the reservoir's (Azerbaijan gascondensate field Bulla-deniz) fluid.

The samples were collected from Azerbaijan gas-condensate field "Bulla-deniz". A general bomb sampler procedure was followed. Gas and liquid samples were taken from the separator gas and liquid outlet lines, respectively. The gas composition was; methane, $91.13 \%$; ethane, $6.78 \%$; propane, $1.1 \%$; butane, $0.58 \%$; pentane, $0.21 \%$; hexane/others $\left(\Sigma \mathrm{C}_{6}\right), 0.04 \%$; carbon dioxide, $0.14 \%$, and nitrogen, $0.02 \%$. This natural gas mixed with nitrogen and carbon dioxide and proportioned. Thus, seven gas mixtures (I-VII) were created which were different from each other Table 1. The condensate composition was; gasoline fraction, $45.9 \%$; aromatic fraction, $17.4 \%$; naphthenic fraction, $36.7 \%$. The condensate density was $748.6 \mathrm{~kg} / \mathrm{m}^{3}$.

Table I Molar compositions of carbon dioxide and nitrogen contained natural gas mixtures

\begin{tabular}{|c|c|c|c|c|c|c|c|}
\hline \multirow[t]{3}{*}{ Gas components } & \multicolumn{7}{|c|}{ Gas mixtures } \\
\hline & $\mathbf{I}$ & II & III & IV & $\mathbf{v}$ & VI & VII \\
\hline & \multicolumn{7}{|c|}{ Molar composition of gas mixtures (\%) } \\
\hline $\mathrm{C}_{1}$ & 91.13 & 82.13 & 73.31 & 58.13 & 82.19 & 72.54 & 64.8 \\
\hline $\mathrm{C}_{2}$ & 6.78 & 5.74 & 4.88 & 1.11 & 6.03 & 5.93 & 4.07 \\
\hline $\mathrm{C}_{3}$ & 1.1 & 1.25 & 1.11 & 0.28 & 1.05 & 0.96 & 0.72 \\
\hline $\mathrm{iC}_{4}$ & 0.32 & 0.2 & 0.16 & 0.18 & 0.25 & 0.21 & 0.17 \\
\hline $\mathrm{nC}_{4}$ & 0.26 & 0.3 & 0.22 & 0.09 & 0.33 & 0.21 & 0.15 \\
\hline $\mathrm{iC}_{5}$ & 0.09 & 0.1 & 0.08 & 0.01 & 0.08 & 0.08 & 0.05 \\
\hline $\mathrm{nC}_{5}$ & 0.12 & 0.07 & 0.06 & 0.02 & 0.06 & 0.05 & 0.04 \\
\hline$\Sigma C_{6}$ & 0.04 & 0.01 & 0.01 & 0.01 & 0.01 & 0.01 & 0.01 \\
\hline $\mathrm{N}_{2}$ & 0.02 & 10.01 & 20.02 & 40 & 0.01 & 0.01 & 0.01 \\
\hline $\mathrm{CO}_{2}$ & 0.14 & 0.19 & 0.15 & 0.17 & 9,99 & 20 & 29.98 \\
\hline
\end{tabular}

The gas-condensate fluids were prepared in the laboratory according to the condensate/gas ratio $\left(200 \mathrm{~g} / \mathrm{m}^{3}\right)$ calculated based on a standard analytical method. ${ }^{1}$ The same natural gas and condensate samples were used for all dedicated experiments to eliminate the impact of the gas-condensate content on the accuracy of the experimental data (error percentage was $\pm 1.2 \%$ ).

\section{Procedure for experimental investigation}

An experimental procedure mainly consists of three steps: i. Gas mixture preparation and investigation.

ii. Loading PVT cell and recombining gas-condensate system.

iii. CCE test and investigation of liquid and gas phases of the gas-condensate system at constant temperature and pressure.

First step: A temperature of $22^{\circ} \mathrm{C}$ was maintained in the experimental units. Natural gas transferred from Module 1 to the Module 5-PVT cell. The PVT cell was purged from the bottom to the top. Samples were 
taken periodically from the PVT cell gas outlet. Sampling continued composition was established in the PVT cell. Furthermore, the physical until the gas composition in the cell confirmed that was identical with and thermodynamic properties of the gas mixture were calculated and the natural gas bottle $( \pm 1.2 \%$ tolerance $)$. The cell pressure then was es- measured at constant temperature $\left(100^{\circ} \mathrm{C}\right)$ and pressure $(12 \mathrm{MPa})$. In tablished at 5MPa, and gas volume was measured. High Pressure Com- total, seven gas mixtures were used for full experimental investigation pressor (HPC) in the Module 3 was loaded by using pure nitrogen or packs. The molar compositions of these $\mathrm{N}_{2}$ and $\mathrm{CO}_{2}$ contained gas mixcarbon dioxide bottle, which was located in the Module 1. Then HPC tures are given in the Table 1. Also, some of the physical and thermo(Module 3) dosed PVT cell (Module 5) and natural gas was gradually dynamic properties Table 2 were calculated by using the method which mixed with $\mathrm{N}_{2}$ or $\mathrm{CO}_{2}$. This process continued until the required gas was recommended in the work. ${ }^{1}$

Table 2 Some of the physical and thermodynamic properties of gas mixtures

\begin{tabular}{|c|c|c|c|c|c|c|c|}
\hline \multirow[t]{3}{*}{ Parameters } & \multicolumn{7}{|c|}{ Gas mixtures } \\
\hline & $\mathbf{I}$ & II & III & IV & $\mathbf{v}$ & VI & VII \\
\hline & \multicolumn{7}{|l|}{ Values } \\
\hline Mass density $\left(\mathrm{kg} / \mathrm{m}^{3}\right)$ & 0.739 & 0.782 & 0.821 & 0.884 & 0.846 & 0.959 & 1.059 \\
\hline Molecular mass $(\mathrm{kg} / \mathrm{kmol})$ & 17.734 & 18.763 & 19.723 & 21.251 & 20.308 & 22.995 & 25.394 \\
\hline Compressibility factor & 0.9976 & 0.9979 & 0.9982 & 0.9988 & 0.9975 & 0.9973 & 0.9971 \\
\hline Critical Temperature (K) & $|89.28|$ & 181.558 & 174.758 & 160.452 & 190.066 & 191.567 & 192.577 \\
\hline Critical Pressure (MPa) & 9.324 & 9.746 & 10.496 & |4.072 & 8.422 & 7.454 & 6.911 \\
\hline
\end{tabular}

Second step: The standard procedure was used ${ }^{1}$ and gas-condensate system was prepared in the PVT cell by recombining the hydrocarbon condensate with the one of the gas mixtures in the Table 1. Condensate $/$ gas ratio was $200 \mathrm{~kg} / \mathrm{m}^{3}$.

Third step: The prepared gas-condensate system was pressurized above the RDP pressure, $30 \mathrm{MPa}$, at $100^{\circ} \mathrm{C}$ as per procedure. The fluid was a single transparent phase that was pale yellow. Then a CCE test was performed to determine the FU, RDP pressures and liquid dropout behaviour at constant temperature. Experimental phenomena for the CCE test were observed through the window of the PVT bomb. This was repeated at least 3 times in the stable thermodynamic condition ensuring a gas-condensate system was recombined correctly (maximum error is $\pm 1.2 \%$ ). After that, the thermodynamic equilibrium conditions were established at this temperature and pressure of $12 \mathrm{MPa}$. At that point, samples were taken from the gas and liquid phases in the constant thermodynamic state. Reason for using these pressure and temperature settings is that, the condition is just above the maximum liquid saturation point.

All experimental equipment was depressurized, vented, drained and purged by nitrogen, thus completing the steps. That meant that the first round of the experiments was completed. Once the equipment was prepared for the next experimental round, the identical procedure was followed but this time a different gas mixture was used for recombining gas-condensate system. The described steps were repeated for all seven gas-condensate systems separately which were combined using the same hydrocarbon condensate but different gas mixture Table 1. It was assumed that these gas mixtures have different solubility capabilities due to the compositions of the $\mathrm{N}_{2}$ and $\mathrm{CO}_{2}$ in the mixtures.

Measurements were repeated when and where possible to increase reliability. Acceptable discrepancies between readings were maintained below $\pm 0.1 \%$.

\section{Experimental results and discussion}

\section{The effect of solubility of gas components on phase transition of gas-condensate systems}

As was described above, the seven gas-condensate systems were investigated. As an example, we provide a description of our observations when we introduced the process at a temperature of $100^{\circ}$ $\mathrm{C}$ during the CCE test. The prepared sample: condensate/gas ratio is $200 \mathrm{~kg} / \mathrm{m}^{3}$; condensate density is $748.6 \mathrm{~kg} / \mathrm{m}^{3}$; gas mixture- I Table 1 was used for recombining gas-condensate system. The system was pressurized to above the RDP pressure, $30 \mathrm{MPa}$, at a temperature of $100^{\circ} \mathrm{C}$ as per procedure. The fluid was a single transparent phase that was pale yellow in colour. As the CCE procedure performed and the pressure dropped (0.1MPa per minute), the colour of the reservoir fluid also changed gradually from pale yellow to pale red. When the pressure was decreased to approximately $21.2 \mathrm{MPa}$, the fluid began to fog up, and the fog thickened with decreasing pressure. The convective phenomena of the thick fog also could be observed by the naked eye through the PVT cell window. With a continuous drop in pressure, a tiny liquid drop precipitated, and the corresponding pressure $(20.1 \mathrm{MPa})$ was then assumed to be the RDP pressure of the investigated gas-condensate fluid. The same condition was observed for all the seven gas-condensate systems, FU and RDP pressures were accordingly recorded. As is shown in the Figure 2, the values of FU and RDP pressures increase according to the increase of $\mathrm{N}_{2}$ and, vice versa, increasing the portion of the $\mathrm{CO}_{2}$ in the gas-condensate fluid decreases the FU and RDP pressures but it increases the difference between FU and RDP pressures Figure 2.

In accordance with the investigation strategy after CCE test, the samples were taken from gas and condensate phases in the constant thermobaric condition (temperature of $100^{\circ} \mathrm{C}$ and pressure of $12 \mathrm{MPa}$ ) which was just above the maximum liquid saturation point. These samples were stabilised - degassed in the atmospheric pressure and 
temperature of $22^{\circ} \mathrm{C}$, and measured the volume of dissolved gas per volume of liquid phase. Figure 3 presents the amount of the dissolved gas per liquid volume on gas-condensate systems with the different compositions as these gas-condensate systems were recombined with different gas mixtures Table 1, In point of fact, results demonstrate that the vaporised volume of condensate in gas phase decreases while the amount of $\mathrm{N}_{2}$ increases in the fluid and, vice versa, the volume of vaporized condensate in gas phase increases if the amount of $\mathrm{CO}_{2}$ increases in the fluid. That could be expected because $\mathrm{CO}_{2}$ is a better "vaporizer" than $\mathrm{N}_{2}$. In this regard, it can be concluded that the amount of vaporised condensate can be increased, even in the same thermobaric conditions, if the solubility feature of gas components of the gas-condensate system is improved Figure 2, Figure 3.

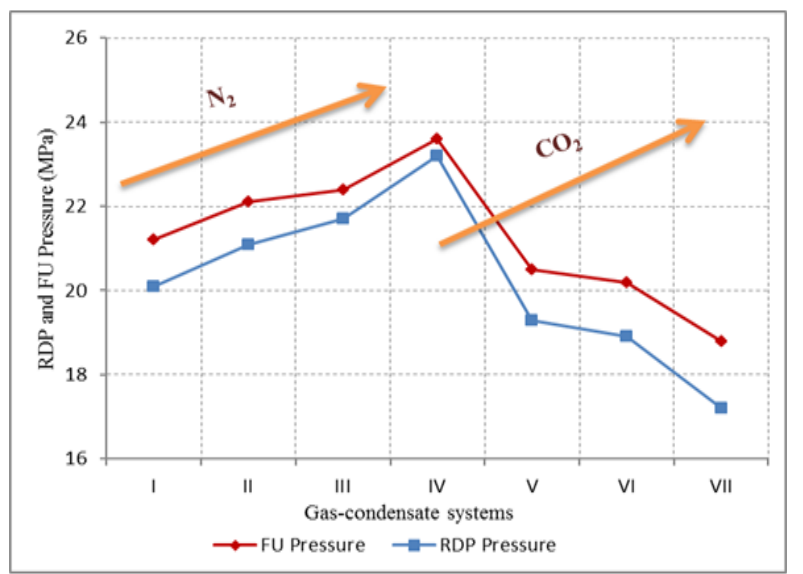

Figure 2 Fog up (FU) and retrograde dew point (RDP) pressures of different gas-condensate systems with different compositions at temperature of $100^{\circ} \mathrm{C}$.

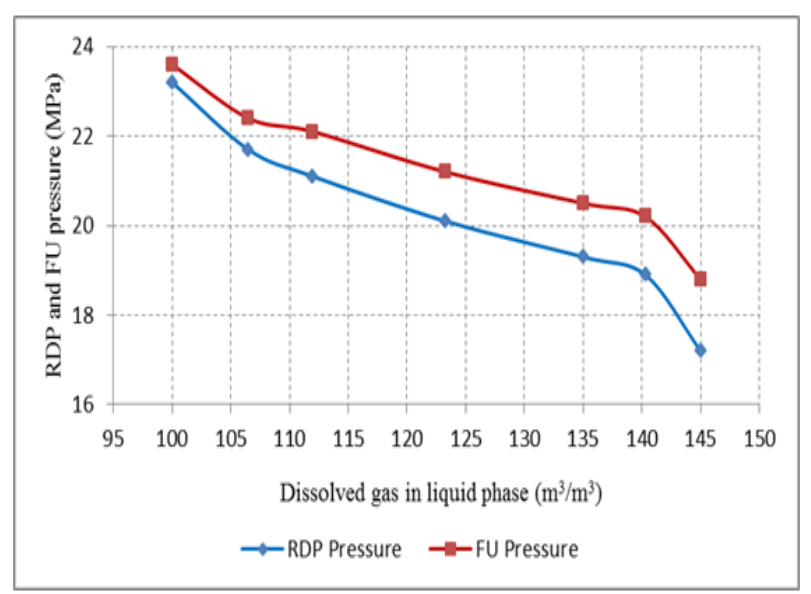

Figure 3 Correlation between FU and RDP pressures and dissolved gas per volume of liquid phase at constant temperature of $100^{\circ} \mathrm{C}$.

As it is described above, a similar experimental phenomenon for the CCE test on gas-condensate systems was observed, including the appearance of the fog condition, which usually is accepted as a common phenomenon for hydrocarbon systems. However, this fog condition is one of the interesting occurrences in the gas-condensate systems. Some of the thermodynamic features of this "phase" are accurately explained by us in the paper. ${ }^{4}$ The state was treated as a colloidal-aerosol system and highlighted some of the physical chemistry aspects. We decided to bring up this specific "phase" or state for discussion again because it could impact on reservoir development and exploitation if the scientific mechanisms are not investigated and understood.

In accordance with this experimental data, the correlation between FU and RDP pressures and dissolved gas per volume of liquid phase at constant temperature was identified. As shown in Figure 3 there is a significant difference between FU and RDP pressures, but improving the solubility of gas components (increasing amount of $\mathrm{CO}_{2}$ in the volume) in the condensate decreases the system $\mathrm{FU}$ and RDP pressures. Also, the difference between FU and RDP pressure increases which means that the stability and the aerosol state of the gas-condensate system improves. In this regard, some of the fundamentals are described in the section below.

\section{The requirements and fundamentals for an effective "vaporiser"}

The role of the Production Technologist is one of achieving optimum performance from the production system and to achieve this, the technologist must understand fully the chemical and physical characteristics of the fluids which are to be produced and, also, the engineering systems which will be utilised to control the efficient and safe production/injection of fluids. In line with this consideration, the physical properties of the gas mixtures were calculated in accordance with acknowledged regression equations which work ${ }^{1}$ presents, for deeper understanding of some basics in this process.

The correlation between condensate/gas ratio of gas phase, critical temperature and pressure of gas mixtures, which were used for recombining of these gas-condensate systems (7 systems) were trended in Figure 4. This experimental data was obtained at the same thermobaric condition $\left(12 \mathrm{MPa}\right.$ and $\left.100^{\circ} \mathrm{C}\right)$ as stated above. Analysis of this data identified that increasing dissolved gas in the liquid phase increases vaporized condensate and that the vaporization capability of gas mixture is elevated (even in the same thermobaric condition) if it's critical temperature increases but critical pressure decreases.

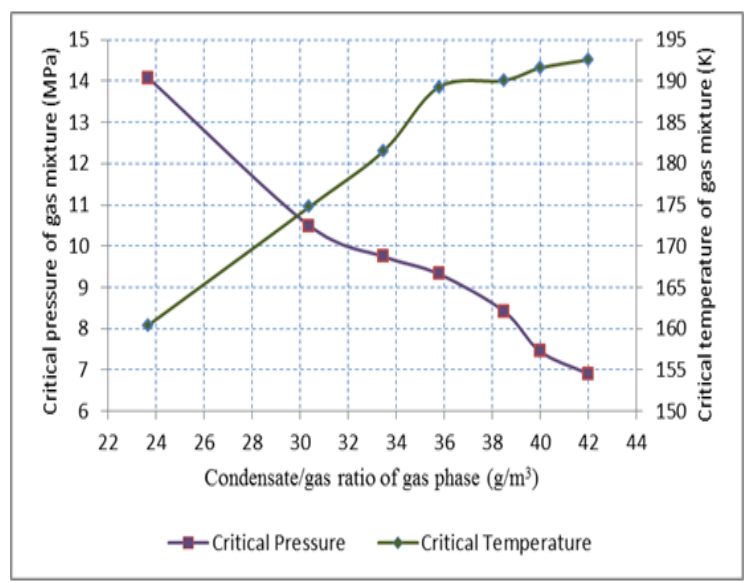

Figure 4 Correlation between condensate/gas ratio of gas phase, critical temperatures and pressures of gas mixtures (which were used for recombination of the gas-condensate systems) at constant temperature of $100^{\circ} \mathrm{C}$ and pressure of $12 \mathrm{Mpa}$.

Correlation between compressibility factors of gas mixtures which were used for recombining gas-condensate systems, condensate/gas ratio of gas phase and dissolved gas in liquid phase were trended at the same thermobaric condition $\left(12 \mathrm{MPa}\right.$ and $\left.100^{\circ} \mathrm{C}\right)$. As it is shown in Figure 5, the condensate/gas ratio of gas phase and dissolved gas in 
the liquid phase increases, even in the same thermobaric condition, if the compressibility factor of gas components decreases.

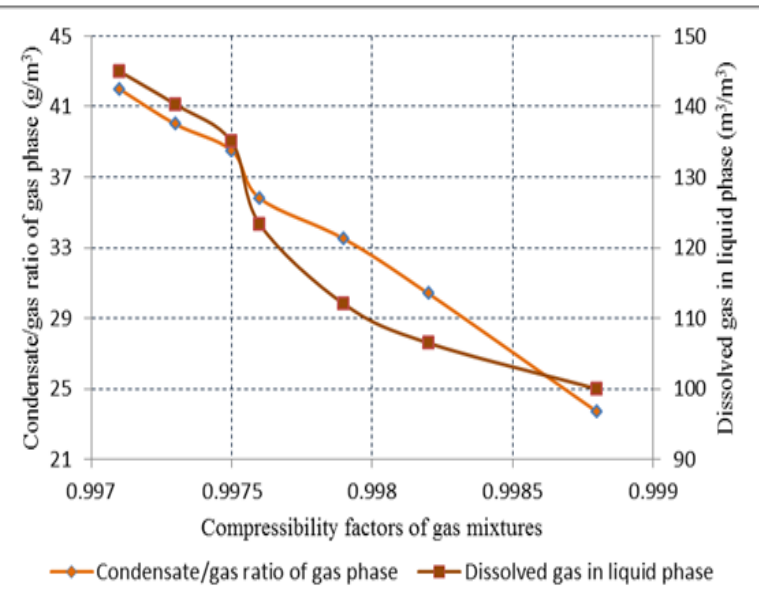

Figure $\mathbf{5}$ Correlation between compressibility factors of gas mixtures (which were used for recombining gas-condensate systems), condensate/gas ratio of gas phase and dissolved gas in liquid phase at same thermobaric condition $\left(12 \mathrm{MPa}\right.$ and $\left.100^{\circ} \mathrm{C}\right)$

In the paper ${ }^{4}$ we examined the process of the liquid or fog forming in the gas-condensate mixture. The fluid was investigated as a colloidal structure in that paper. The physical and thermodynamic features of this structure were explained based on the properties of colloidal systems. It is known that microscopic particles of one phase dispersed in another are generally called colloidal solutions or dispersions. These are also known as the colloidal dispersions because the substances remain dispersed and do not settle to the bottom of the container. We can accept that a gas-condensate system is in a colloidal-dispersion condition when it is in retrograde condensation/ vaporization zone. In this case, the gas components are the dispersion medium and the condensate is the dispersed phase in the system.

Now, we can explain why the FU and DP pressures decrease and the difference between these pressures become larger with the increase of solubility gas components. At the same time the amount of the vaporised or dispersed liquid components increases. This finding expresses that the stability of the aerosol state of the gas-condensate improves by increasing the solubility of the gas components. The stability of a colloidal system is defined by particles remaining suspended in solution at equilibrium. Stability is hindered by aggregation and sedimentation phenomena, which are driven by the colloids' tendency to reduce surface energy. Reducing the interfacial tension will stabilize the colloidal system by reducing this driving force. In a gas-condensate system, condensation can occur when the aerosol concentration is sufficient to cause the coalescing of fine condensate particles, and densities of the individual particles are significantly greater than the gas phase. The opposite situation will occur with increasing critical temperature and decreasing compressibility factor of gas components. Therefore, injected gas for gas cycling or re-vaporization of condensate from the core can be controlled for the purpose of increasing its solubility and condensatebearing capability accordingly.

\section{Conclusion}

Based on the obtained results from the CCE test of seven different gas-condensate systems and analysis of the samples taken under the same thermobaric conditions from liquid and gas phases specified that, improving the solubility of gas components in the condensate decreases the system FU and RDP pressures. Also, the difference between FU and RDP pressure increases which means that the stability of the aerosol state of the gas-condensate system improves.

The correlation between condensate/gas ratio of gas phase, the critical temperature and pressure of gas mixtures, which were used for recombining of different gas-condensate systems, were investigated. Analysis of this data identified that increasing dissolved gas in the liquid phase, increases vaporized condensate and vaporization capability of gas mixture is elevated (even in same thermobaric condition) if it's critical temperature increases but critical pressure decreases.

The correlation between compressibility factors of gas mixtures which were used for recombining gas-condensate systems, condensate/gas ratio of gas phase and dissolved gas in liquid phase was considered. It was shown that the condensate/gas ratio of the gas phase and dissolved gas in the liquid phase increases, even in the same thermobaric condition, if compressibility factor of gas components decreases.

Experimental data demonstrates that the vaporised volume of condensate in gas phase decreases while the volume of $\mathrm{N}_{2}$ increases in the fluid. Conversely, the volume of vaporized condensate increases if $\mathrm{CO}_{2}$ increases in the fluid. The reason for this is that the solubility of $\mathrm{CO}_{2}$ is better than $\mathrm{N}_{2}$. This finding expresses that the stability of the aerosol state of the gas-condensate system improves by increasing the solubility of the gas components. That is why $\mathrm{CO}_{2}$ is recognised as a good "vaporizer".

\section{Acknowledgements}

None.

\section{Conflict of interest}

The author declares no conflict of interest.

\section{References}

1. MT Abasov, ZY Abbasov, VM Fataliyev, et al. Applied thermodynamic aspects in oil and gas production. Nafta Press, Baku, Azerbaijan, 2013.

2. T Takeda, Y Fujinaga, K Fujita. Fluid Behaviours around a Well in Gas-Condensate Reservoirs. SPE Asia Pacific Oil and Gas Conference and Exhibition, Kuala Lumpur, Malaysia, 1997.

3. FB Thomas, DB Bennion, G Andersen. Gas Condensate Reservoir Performance. Petroleum Society of Canada. Journal of Canadian Petroleum Technology. 2009;48(7):18-24.

4. ZY Abbasov, VM Fataliyev. The effect of gas-condensate reservoir depletion stages on gas injection and the importance of the aerosol state of fluids in this process. Journal of Natural Gas Sciences and Engineering. 2016;31:779-790.

5. A Kh Mirzadzhanzade, OL Kuznetsov, KS Basniev, et al. Osnovy tekhnologii dobychi gaza (Foundation of gas recovery technology), M Nedra, Russian, 2003

6. A Mehriban, F Crespo. A New Multi-Sample EOS Model for the Gas Condensate Phase Behavior Analysis. Oil \& Gas Science and Technology. 2004;66(6):1025-1033.

7. L Du, JG Walker, GA Pope, et al. Use of Solvents to Improve the Productivity of Gas Condensate Wells. SPE Annual Technical Conference and Exhibition. Texas, Dallas, USA, 2000. 
8. HA Al-Abazi, MM Sharma, GA Pope. Revaporization of condensate with methane flood. Society of Petroleum Engineers, Texas, Houston, USA, 2004.

9. S Li, X Zheng, Z Dia, et al. Investigation of revaporizotion of retrograde condensate. Society of Petroleum Engineers, Manama, Bahrain, 2001.

10. AKM Jamaluddin, S Ye, J Thomas, et al. Experimental and Theoretica Assessment of Using Propane to Remediate Liquid Build-up in Condensate Reservoirs Paper. SPE Annual Technical Conference and Exhibition, New Orleans, Louisiana, USA, 2001.

11. MT Abasov, ZY Abbasov, VM Fataliyev, et al. A New Phenomenon in Phase Transformations of Gas-Condensate Systems and Its Experimental Study. Doklady Earth Sciences of Russian Academy of Sciences. 2005;403(6):858-860.
12. K Jessen, FM Orr. Gas Cycling and the Development of Miscibility in Condensate Reservoirs. SPE Reservoir Evaluation \& Engineering. 2004;7(5):334-341.

13. SE Eshraghi, MR Rasael, S Zendehboudi. Optimization of miscible $\mathrm{CO}_{2}$ EOR and storage using heuristic methods combined with capacitance/ resistance and Gentil fractional flow models. Journal of Natural Gas Sciences and Engineering. 2016;32:304-318.

14. NN Hamidov, VM Fataliyev. Influence of solubility of the different composition gases in the hydrocarbon condensate to the production parameters of gas condensate fields. SOCAR Proceedings. 2015;4:36-40.

15. NN Hamidov, VM Fataliyev. Experimental study into the effectiveness of the partial gas cycling process in the gas-condensate reservoir development. Petroleum Science and Technology. 2016;34(7):677-684. 